Mappemonde

Revue trimestrielle sur l'image géographique et les formes du territoire

$131 \mid 2021$

Varia

\title{
Un week-end marathon pour un mapathon
}

\section{Abdou Diouck}

\section{OpenEdition}

Journals

Édition électronique

URL : https://journals.openedition.org/mappemonde/6290

DOI : $10.4000 /$ mappemonde.6290

ISSN : 1769-7298

Éditeur

UMR ESPACE

\section{Référence électronique}

Abdou Diouck, " Un week-end marathon pour un mapathon », Mappemonde [En ligne], 131 | 2021, mis

en ligne le 08 juillet 2021, consulté le 14 juillet 2021. URL : http://journals.openedition.org/

mappemonde/6290 ; DOI : https://doi.org/10.4000/mappemonde.6290

Ce document a été généré automatiquement le 14 juillet 2021.

\section{(c) (i) (2) (2)}

La revue Mappemonde est mise à disposition selon les termes de la Licence Creative Commons Attribution - Pas d'Utilisation Commerciale - Partage dans les Mêmes Conditions 4.0 International. 


\title{
Un week-end marathon pour un mapathon
}

\author{
Abdou Diouck
}

1 La représentation spatiale des flux n'est pas une tâche triviale et rend parfois difficile la compréhension ou la lecture d'une carte. Contrairement aux cartes en aplat de couleurs (motifs, agrégation de flux, nébuleuse), le webmapping s'impose comme la référence et bouleverse les modes de représentations. Il offre d'énormes possibilités à l'utilisateur : un espace convivial et ludique pour interagir avec les différentes composantes de la carte.

2 Des applications comme, entre autres, Kepler, Mapbox, Arabesque rendent possible cette représentation. Mais, au-delà d'un certain nombre de flux, les cartes deviennent illisibles. Pour s'affranchir de ces inconvénients, le Mapathon, concours de (géo)datavisualisation, proposé dans le cadre du colloque Tous (im)mobiles, tous cartographes? (cartomob.sciencesconf.org), était un terrain idéal pour se lancer dans une nouvelle expérimentation. J'ai voulu explorer ici une nouvelle façon de visualiser les flux.

3 Comment cartographier le transport aérien en France métropolitaine de 1999 à 2020 ? Comme nous l'avons $\mathrm{vu}$, aucune réponse n'est simple, synonyme de consensus. Nous disposons de deux jeux de données :

- le trafic de passagers et de marchandises des liaisons entre villes aéroportuaires (plus de 5000 passagers/an);

- les données Eurostat 2010-2020 sur les fréquentations (nombre de passagers, de marchandises [fret+courrier] et de mouvements d'avions) par ville et par mois.

4 Pour répondre à ces deux objectifs, mon choix s'est tourné naturellement vers le développement d'une application cartographique, le meilleur moyen pour une représentation spatio-temporelle. 
Figure 1. Trafic de passagers et de marchandises des liaisons entre villes aéroportuaires (exemple : Lyon)

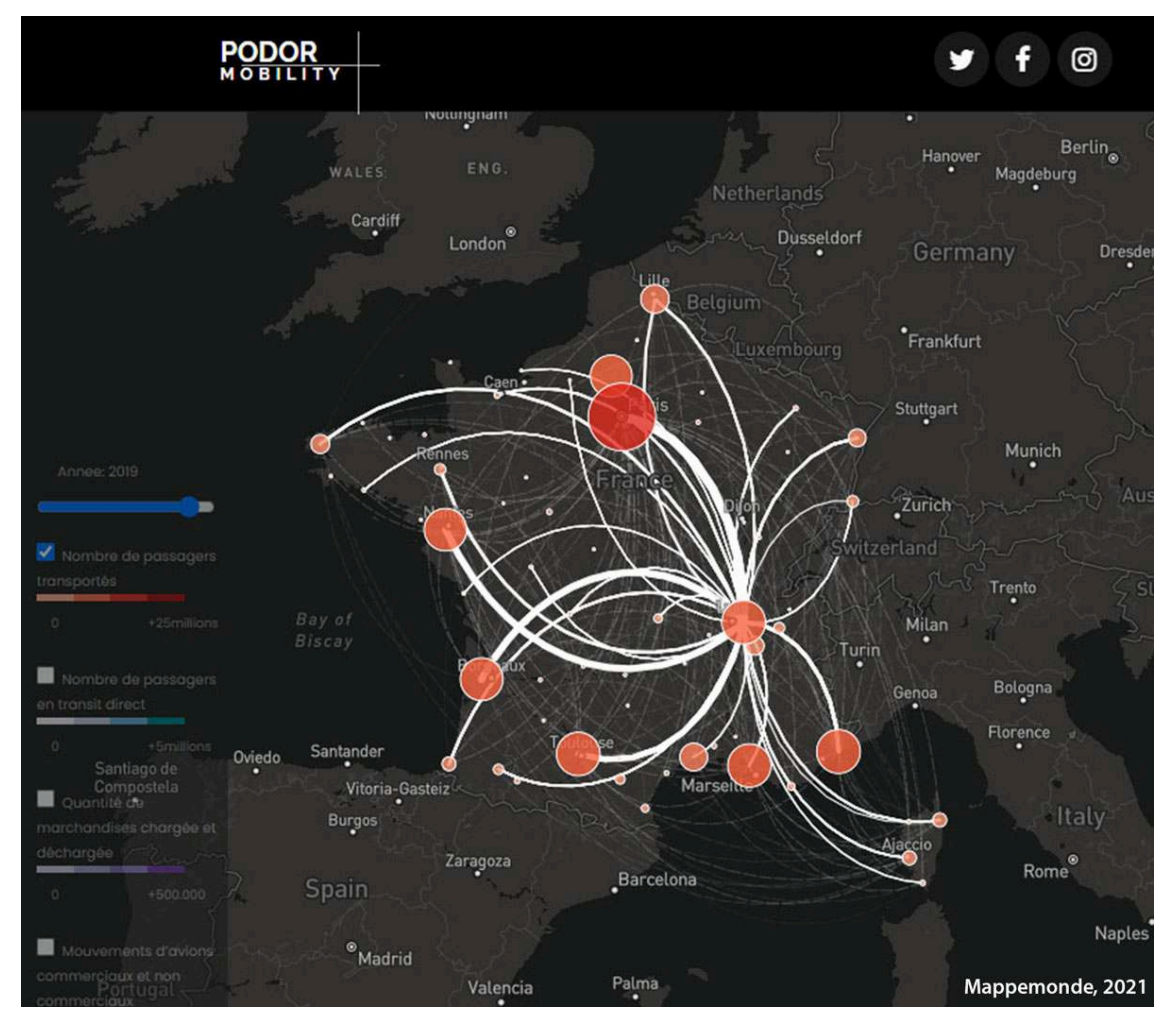

5 L'application présente une page synthétique (figure 1), qui résume le trafic de passagers et de marchandises des liaisons entre villes aéroportuaires (répartition des passagers par aéroport, nombre de passagers, de marchandises et de mouvements d'avion, classements...). Cette présentation permet à l'utilisateur d'avoir un aperçu des évolutions spatio-temporelles des flux. Pour une meilleure expérience de l'utilisateur, j'ai fait également le choix de ne présenter les flux qu'au survol des aéroports pour ne pas surcharger la carte. Cela facilite la visualisation des indicateurs dans le temps et l'espace.

6 La légende propose une liste d'indicateurs permettant d'interagir avec la carte :

Nombre de passagers transportés ;

Nombre de passagers en transit direct ;

Quantité de marchandises chargée et déchargée ;

Mouvements d'avions commerciaux et non commerciaux.

7 En cliquant sur un aéroport, l'utilisateur peut accéder aux données de fréquentation (nombre de passagers et de marchandises). J'ai utilisé ici les données statistiques associées des nœuds pour générer une fiche synthétique pour chaque aéroport. En complément («Cliquer ici pour plus de détails»), j’ai également proposé des statistiques détaillées pour chaque liaison (figure 2). 
Figure 2. Répartition spatio-temporelle du trafic aérien (exemple : Lyon)

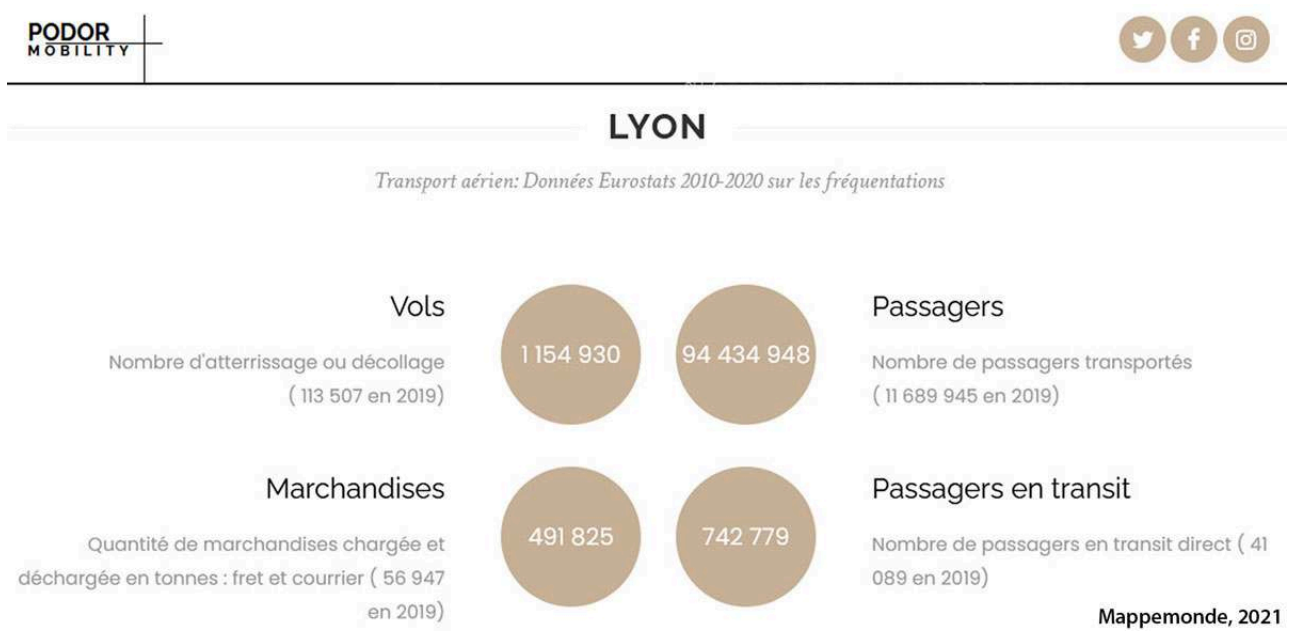

8 À la manière des storymap, en naviguant vers le bas (scrolling ou bouton situé en bas à droite de l'interface), l'utilisateur retrouve un ensemble de visualisations synthétiques sur les données de fréquentation pour une ville donnée ou sur l'ensemble du réseau (figure 3) :

répartition des passagers par aéroport en 2019 ;

nombre d'atterrissages/décollages, de passages, passagers en transit direct, tonnes de marchandise (nombre de vols, passagers, trafic, répartition et évolution des fréquentations).

Figure 3. Trafic aérien par aéroport (exemple : Lyon)

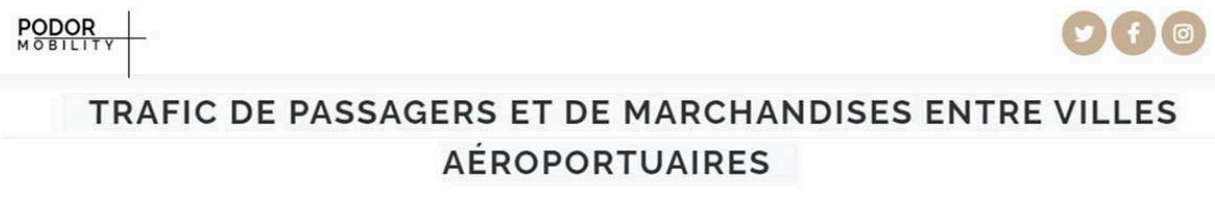

Répartition des passagers par aéroport en 2019

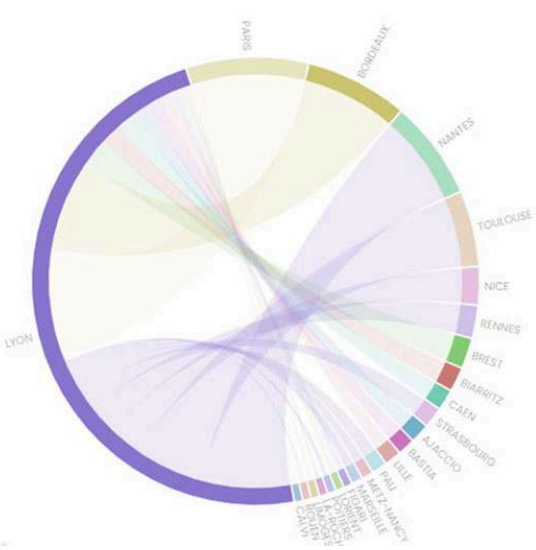

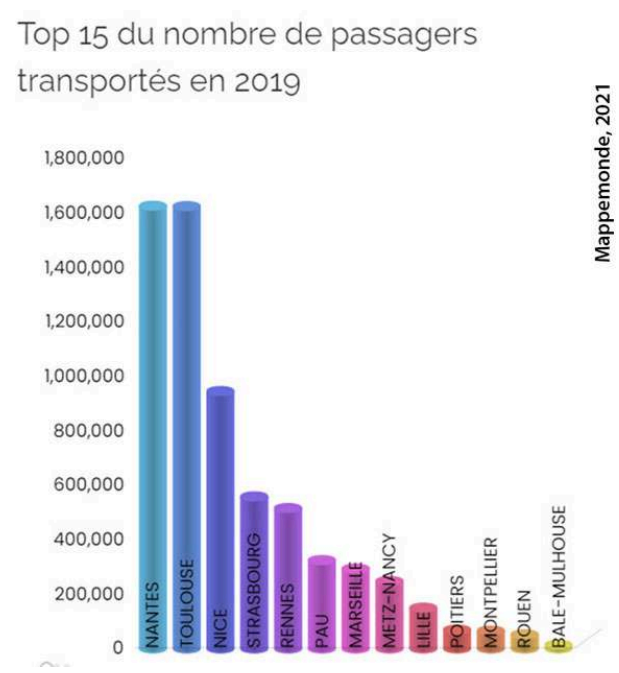

Pour la réalisation de cette application, j'ai choisi de travailler uniquement avec des outils open source. Coté applicatif, j'utilise Laravel, un framework web open source, écrit 
en PHP, respectant le principe modèle-vue-contrôleur et entièrement développé en programmation orientée objet. Laravel est distribué sous licence MIT, avec ses sources hébergées sur GitHub. Le projet réalisé dans le cadre de ce mapathon est d'ailleurs accessible sur mon compte github (https://github.com/diouck/podor).

Sur la partie «données », et pour la création d'arcs fluides, je me suis naturellement tourné vers Postgis, une extension spatiale pour PostgreSQL. L'avantage de celle-ci est de pouvoir gérer toute la chaîne de traitements en seulement quelques requêtes.

11 Un exemple pour les géomaticiens : pour générer les arcs, il est possible d'utiliser la fonction ST_CurveToLine en complément de la fonction ST_OffsetCurve pour calculer le décalage $-1 / 10^{e}$ de la longueur de la ligne d'origine. Voici le SQL que j'ai utilisé pour générer les lignes courbes à partir des lignes droites d'origine :

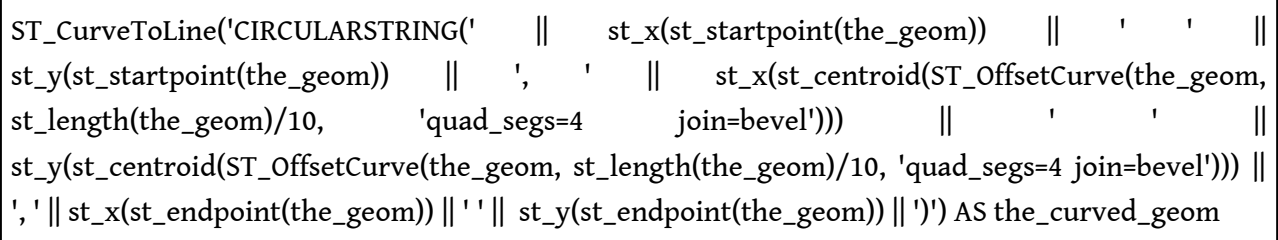

12 Au final, l'application est ergonomique et responsive design grâce à un template open source téléchargé sur colorlib associé à Laravel. Le rendu cartographique est assuré par Mapbox, ce qui rend la visualisation encore plus esthétique, contrairement à ce que l'on a l'habitude de voir.

13 Travailler à la fois sur les données et sur la partie applicative fut une expérience personnelle très enrichissante. Le défi était, pour moi, de proposer une visualisation uniquement avec des outils open source. Comme stipulé plus haut, la principale difficulté que j'ai pu rencontrer, au-delà du temps imparti, était la bidirectionnalité des flux. Il m'a donc fallu adapter les traitements pour faciliter la représentation.

14 Je tiens à remercier l'équipe des organisateurs de m'avoir offert cette opportunité ainsi que tous les participants qui ont fourni des propositions variées et de qualité.

\section{AUTEUR}

\section{ABDOU DIOUCK}

GéoAfrica, plateforme dédiée à l'information géographique (https://geoafrica.fr/) 